\title{
Significados y vulnerabilidad social ante el terremoto y tsuna- mi del 27 de febrero del 2010: la dimensión subjetiva desde los "damnificados"
}

\section{Meanings and social vulnerability to 27F earthquake and tsunami: subjec- tive dimension of Chilean victims}

\author{
José Sebastián Sandoval Diaz* \\ Universidad de Atacama, Chile \\ Daniela Fava Callejas \\ Universidad de Chile \\ (Rec.: septiembre de 2016 - Acept.: noviembre de 2016)
}

\begin{abstract}
Resumen
El presente estudio tuvo como objetivo conocer los significados y experiencias de 14 personas damnificadas por el desastre del $27 \mathrm{~F}$ pertenecientes a la comuna de Constitución, Chile. Para esto se utilizó un diseño cualitativo de corte fenomenológico. Para la selección de participantes se empleó un muestreo intencionado. En cuanto a la producción de datos, se aplicó la técnica de entrevistas en profundidad cuyo producto fue analizado con la estrategia de codificación de la teoría fundamentada. Los resultados se dividen en dos temas principales: a) nos quedamos con lo puesto: (sobre)viviendo en la incertidumbre; y b) desorganización institucional vs. apoyo civil. Se identificaron subcategorías en cada tema de análisis, las cuales son ilustradas por los datos narrativos. Asimismo, se discuten y valoran las implicancias en la incorporación de la subjetividad de los afectados al proceso de comprensión psicosocial en la investigación sobre los desastres socio-naturales.
\end{abstract}

Palabras clave: subjetividad, vulnerabilidad social, desastres socio-naturales, terremoto, Chile.

\begin{abstract}
The present study aimed to understand meanings and shared experiences of 14 persons from Constitución (Chile) affected by the natural disaster on 27 February (27F). In order to achieve this goal, phenomenological qualitative design was used, selecting a purposive sampling comprised of people affected by the $27 \mathrm{~F}$ earthquake and tsunami. Qualitative data collection was produced via in-depth interviews which were analyzed through strategies of codification based on grounded theory. The results are split into two main subjects: A) We lost everything: Surviving under uncertain conditions. B) Institutional disorganization vs. civil society. Five subcategories were identified inside each category of analysis and are illustrated by their narrative stories. Additionally, implications connected with the incorporation of that subjectivity experienced by victims to the process of psychosocial understanding (framed into the research of socio-natural disaster) is discussed and assessed.
\end{abstract}

Keywords: subjectivity, social vulnerability, socio-natural disaster, earthquake, Chile.

\footnotetext{
* Correspondencia a: José Sandoval Díaz. Departamento de Psicología, Universidad de Atacama, Copayapu, 485, Copiapo, Chile. E-mail: jose.sandoval@uda.cl
} 


\section{Introducción}

En la actualidad, los desastres de origen natural y antrópico se han convertido en uno de los principales obstáculos en el desarrollo económico y social tanto de países como de los medios de vida comunitarios y familiares (Programa de las Naciones Unidas para el Desarrollo [PNUD], 2014). Se estima que el costo total de este tipo de eventos ascendió a US\$3800 billones entre 1980 y 2012 a escala global. A raíz de esto, comunidades y familias en condiciones de pobreza estructural tienden a presentar una baja capacidad para anticipar, resistir y recuperarse ante el impacto de este tipo de eventos (Banco Mundial, 2014).

Respecto a la situación de Chile, las características geográficas en las cuales el territorio se emplaza sumadas a la vulnerabilidad social construida, la recurrencia de amenazas naturales y los efectos devastadores que estas conllevan han sido la tónica constante a lo largo de la historia nacional. Basta mencionar la presencia de 25 terremotos en los últimos 70 años, 13 sequías en las últimas 5 décadas, 8 tsunamis en los últimos 90 años, 28 erupciones volcánicas en el último siglo, así como también otras tantas marejadas, nevadas, incendios forestales, inundaciones y aluviones (Oficina Nacional de Emergencia del Ministerio del Interior y Seguridad Pública [ONEMI], 2014). Solo por mencionar algunos de estos eventos sucedidos en las últimas décadas, tenemos la erupción del volcán Chaitén (2008), el terremoto y tsunami en la zona centro-sur denominado 27F (2010), el terremoto del norte grande (2014), el incendio antrópico de Valparaíso (2014), los aluviones e inundaciones en las regiones de Antofagasta, Atacama y Coquimbo (2015), así como también la activación de los volcanes Villarrica y Calbuco (ONEMI, 2014).

A pesar de que la mencionada tipología de amenazas naturales es variada, los fenómenos que han manifestado mayor presencia, frecuencia y severidad en cuanto a su impacto han sido los terremotos (Banco Interamericano de Desarrollo [BID], 2015). Solo cuantificando las pérdidas económicas asociadas al terremoto y posterior tsunami del 27 de febrero del 2010, estas ascendieron, aproximadamente, a los US $\$ 30.000$ millones, equivalentes al $15 \%$ del PIB nacional (Comisión Económica para América Latina y el Caribe [CEPAL], 2010). Sin embargo, si bien el análisis económico y material de las pérdidas se torna un elemento necesario para la evaluación de daños frente a este tipo de eventos, este elemento por sí solo se torna insuficiente para una comprensión holística y compleja del impacto psicosocial tanto en lo relativo a sus dimensiones sociales como simbólicas post evento.

\section{Marco teórico}

En la problematización sobre riesgos y desastres socio-naturales, desde las ciencias sociales, la dimensión de la vulnerabilidad social ha ido adquiriendo paulatina relevancia. Esto ha permitido la explicación no solo de los efectos económico-sociales que dejan a su paso los desastres, sino también de las condiciones causales previas de susceptibilidad/exposición al peligro para comunidades, familias y personas ante fenómenos de origen natural o antrópico (Maskrey, 1993). Esta exposición al peligro a lo largo de los territorios habitados y apropiados se expresa diferencialmente tanto en la exposición física-espacial a las amenazas como también en la susceptibilidad y capacidades movilizadas; sean estas económicas, organizacionales y motivacionales. Esta variabilidad en presencia y grado de las capacidades mencionadas se vincula no solo al acceso y uso del recurso movilizado ante las estructuras de oportunidades del entorno, sino también a la dimensión temporal del riesgo/desastre en la cual es movilizada; sea esta frente la anticipación, respuesta y recuperación post evento.

Sin embargo, en la actualidad existe una falta de consenso respecto a la definición, medición y evaluación de la vulnerabilidad ante este tipo de eventos. Esto se aprecia en las múltiples disciplinas, áreas, profesiones y ciencias aplicadas que se abocan a su estudio, las cuales priorizan distintas dimensiones, temporalidades y unidades de análisis (Weichselgartner, 2001; Wisner, 2016). En este mismo sentido Allan Lavell (2004) ha expresado "la pérdida paulatina de especificidad en el uso del término al tratar de definir un conjunto de cosas tan disimiles, que conceptual, teórica y prácticamente perdió su rumbo y seriedad" (Lavell, 2004). Para Oliver-Smith (2007) esta noción debiese ser el nexo conceptual que dé cuenta de la multidimesionalidad de los riesgos y desastres socio-naturales, a partir de la relación entre sociedad, medioambiente, Estado y mercado.

A partir de este carácter multidimensional del término, se pueden encontrar múltiples definiciones. Cardona (2003), por ejemplo, lo define como el grado de exposición de los ecosistemas y la susceptibilidad de grupos humanos ante un estrés particular de tipo natural (Romero \& Vidal, 2015). Para organismos internacionales como la Organización de Naciones Unidas (ONU), de acuerdo al Informe de Desarrollo Humano Mundial 2014 titulado: "Sostener el progreso humano: Reducir Vulnerabilidades y Construir Resiliencia" la noción es definida realizando un fuerte hincapié en su vínculo con el desarrollo humano, al introducir la noción de vulnerabilidad humana entendida como el potencial grado de erosión de capacidades, oportunidades y opciones de las personas ante una crisis (PNUD, 2014). Desde este enfoque se torna necesario fortalecer la resiliencia humana, con el fin de asegurar, de forma sólida y sostenible, las oportunidades y capacidades sociales alcanzadas.

Para el caso de las regiones subdesarrolladas, como es 
el caso de América Latina, Cardona (1993) considera que la vulnerabilidad social sería la causa de fondo de la fragilidad técnica-espacial e institucional, en las etapas tanto de preparación y respuesta como de recuperación frente este tipo de eventos. Por consiguiente, a diferencia de la amenaza natural, la cual actúa como detonante contingente, la vulnerabilidad social sería una condición construida y dinámica, ligada íntimamente a las dimensiones socioculturales del desarrollo y bienestar poblacional (Maskrey, 1989). Sin embargo, estas distintas definiciones han tendido a concebir una mirada "pasiva" de la vulnerabilidad (Sandoval et al., 2015), es decir, comprendiéndola solo bajo los términos de exposición o susceptibilidad estructural de daño, relegando a una posición predominantemente pasiva e inactiva a la sociedad civil. Por lo tanto, sin negar las dimensiones estructurales/procesuales de la vulnerabilidad social a la base, así como de la exposición física-espacial frente a distintos tipos de amenaza, se torna necesario (re)considerar e incorporar en esta definición las diversas disposiciones, acciones y prácticas humanas que reproducen, resisten y mitigan tanto las causas como las consecuencias de este tipo de eventos. Bajo este marco, Anderson y Woodrow (1989) no solo identifican y caracterizan tres tipos diferenciados de vulnerabilidad: a) física-material, b) social-organizacional y c) motivacional-actitudinal, sino que a su vez incorporan una dimensión agencial de recursos y fortalezas comunitarias ante este tipo de eventos bajo la noción de capacidad. Para los mencionados autores, esta mirada determinística de las causas estructurales de la vulnerabilidad sobre la acción social ha tendido a (re)producir una mirada pasiva de quienes vivencian directa o indirectamente este tipo de eventos. Un ejemplo de esto lo constituye el término "damnificado", el cual concibe al sujeto como afectado en lo material o en su salud mental, soslayando sus recursos, experiencias y tácticas individuales y colectivas para afrontar este tipo de situaciones. Sin embargo, esta (re) visualización de un sujeto activo comprendido tanto en términos colectivos como singulares no debe ser abstraída de las condiciones dinámicas y socioculturales de la vulnerabilidad social de base, la cual limita y condiciona la configuración subjetiva en su accionar ante este tipo de eventos (Sandoval et al., 2015). Por lo tanto, nociones tales como capacidad y resiliencia humana han ido adquiriendo importancia como eje comprensivo respecto a los posibles estilos de afrontamientos durante este tipo de eventos (Pelling, 2010).

Las investigaciones que consideran la perspectiva de los propios sujetos frente a los desastres se han centrado, convencionalmente, en la percepción del riesgo, el impacto psicosocial en la salud mental y el comportamiento de las poblaciones en riesgo. No obstante, otros enfoques actuales han explorado el papel de la subjetividad o consciencia de los propios sujetos en riesgo en la comprensión de sus propias causas de vulnerabilidad, y en algunos caso, incluso, en la resistencia que estos presentan hacia la ayuda de las autoridades (Wisner, 2016).

Respecto a esta relación entre subjetividad y acción, a la luz del rol activo del sujeto frente a los desastres, Pérez, Agurto y Arteaga (2012) han acuñado el término vulnerabilidad social subjetiva, la cual es definida como la "situación de propensión al daño que es facilitada por procesos de significación, construidos culturalmente por los sujetos, en relación a sí mismo y a su entorno, en un contexto socio-histórico determinado por el desastre" (p. 8).

Desde esta dimensión se plantea que las personas toman decisiones sin hacer uso de todos los recursos y oportunidades que podrían aprovechar, ni ordenan sus acciones racional y causalmente; más bien conducen un entramado de acciones que se basan en distintos principios, sumando incluso nuevas tensiones y conflictos a los ya presentes (Arteaga et al., 2015).

A partir de los antecedentes presentados, el propósito del presente estudio de tipo fenomenológico es conocer los significados y experiencias compartidas de personas vulneradas por el desastre del $27 \mathrm{~F}$ pertenecientes a la comuna de Constitución. La presente investigación toma la noción de significado propuesta por Kenneth Gergen, quien la define como una construcción relacional que necesita de acciones y suplementos para ser contextualizada, resaltando la importancia del sentido como elemento mediacional (Mendoza, Ramos, Ortiz \& Jaramillo, 2010). Por último, desde la dimensión subjetiva del desastre, esta investigación busca conocer el significado sentido desde la propia experiencia de aquellos que han sido rotulados como "damnificados", permitiendo obtener un conocimiento más profundo de sus disposiciones y valoraciones de las acciones desplegadas ante este tipo de eventos. $A$ su vez, el aporte de la presente investigación se vincula al potencial reconocimiento e incorporación de la dimensión subjetiva de la vulnerabilidad social configurada estructuralmente, permitiendo la ampliación tanto teórica como práctica de dicho concepto en pos del desarrollo de la gestión y reducción integral de los riesgos ante desastres socio-naturales (RRD).

A continuación, a modo de caracterización, se presentan algunos antecedentes vinculados al contexto del caso de estudio.

\section{El contexto: Constitución en el 27F}

En la madrugada del 27 de febrero del año 2010, un terremoto de 8,8 en la escala de Richter, acompañado de un posterior tsunami, impactó en la zona centro-sur de Chile1. El terremoto devastó parcialmente

\footnotetext{
${ }^{1}$ El día 27 de febrero a las 03:34 horas, hora local, se registró un terremoto de magnitud 8,8 en la escala MW que tuvo duración aproximada de tres minutos. El epicentro se situó en la costa de la región del Mau-
} 
cinco grandes ciudades, cuarenta y cinco ciudades de tamaño medio y más de 900 aldeas rurales (Bresciani, 2010). Constitución ${ }^{2}$, una ciudad costera de la región del Maule, fue una de las comunas con mayor daño producto del desastre. Mientras que el terremoto devastó la mayor parte de sus fabricaciones de adobe y edificios históricos de la ciudad, el posterior tsunami destruyo gran parte de las viviendas ubicadas a la orilla del rio (Tironi, 2014). De acuerdo a datos del Programa de Reconstrucción Sustentable de Constitución (como se citó en Tironi, 2014), un total 100 personas murieron en la zona, 1789 viviendas fueron parcialmente destruidas, 1200 sufrieron daños irreparables, y alrededor del 80 por ciento del centro de la ciudad sufrió algún tipo de daño.

\section{Metodología}

La presente investigación cualitativa utilizó un diseño de investigación fenomenológico cuyo propósito principal es explorar, describir y comprender los significados y experiencias compartidas de las personas respecto a un fenómeno (Flick, 2007). En este caso, nos referimos a la vivencia subjetiva ante el desastre del 27F. El enfoque fenomenológico adoptado se adscribe a la hermenéutica, centrándose en la interpretación, por parte del investigador, sobre el basto "texto" de la experiencia humana a relevar (Creswell, Hanson, Plano \& Morales, 2007).

\section{Selección de contextos y participantes}

La presente investigación utilizó un muestreo intencionado, procediendo "según la relevancia de los casos, en lugar de hacerlo por su representatividad" (Flick, 2007, p. 80). En primer lugar, se utilizó una selección estratégica del contexto, priorizando para esto los criterios de accesibilidad y de homogeneidad frente al impacto del desastre (Valles, 2000), seleccionando, para este caso, el sector de la poza en Constitución.

Los participantes de esta investigación fueron 14 personas caracterizadas como afectadas, las cuales debieron cumplir los siguientes criterios de inclusión muestral:

- Que presentaran daño parcial o total de su vivienda

- Que hubiesen sido clasificados como damnificados por parte de los organismos gubernamentales

- Que residieran en Constitución a los menos desde

le, aproximadamente a $8 \mathrm{~km}$ al oeste de Curanipe y $115 \mathrm{~km}$ al noreste de Concepción. Según la agencia científica United States Geological Survey (USGS), el evento ocurrió en la frontera de las placas tectónicas Nazca y Suramericana, debido a una solapamiento en la interfaz de ambas, con la primera terminando por debajo de la segunda.

2 Según datos poblacionales del Instituto Nacional de Estadísticas (INE), al año 2002, esta comuna cuenta con una población de 46.081 habitantes, de los cuales un $18 \%$ se encontraba en situación de pobreza (Ministerio de Planificación, 2011). febrero del 2009 a la fecha de la realización de la investigación (Enero del 2015).

\section{Estrategia de producción de datos}

Como técnica de producción de información se utilizó la entrevista en profundidad, la cual se define como los "reiterados encuentros cara a cara entre el investigador y los informantes, encuentros dirigidos hacia la comprensión de las perspectivas que tienen los informantes respecto de sus vidas, experiencias o situaciones, tal como lo expresan con sus propias palabras" (Taylor \& Bogdan, 1998, p. 101). En la construcción de la entrevista se incorporaron temáticas ordenadas temporalmente, las cuales se enfocaron en las vivencias y acciones desplegadas previamente, durante y posteriormente al desastre.

\section{Procedimientos}

El trabajo de campo se realizó entre fines del año 2014 y principios del 2015. La selección de contextos a través de los cuales se contactaron a los participantes siguió dos criterios. El primero consistió en que los contextos hubiesen sido afectados parcial o totalmente por el desastre desde el punto de vista físico-material. El segundo criterio radicó en que se permitiese el acceso frecuente de los investigadores, esto es, que los contextos no estuviesen alejados ni cerrados. Los participantes fueron contactados en sus propios hogares e incorporados al estudio previo cumplimiento de los criterios de inclusión muestral. En cuanto a la producción de datos, se realizó en el hogar de los participantes. Los relatos fueron audiograbados y transcritos íntegramente por los autores. Posteriormente se realizó el proceso de codificación mediante el modelo analítico de la teoría fundamentada (Strauss \& Corbin, 2002). La información fue analizada siguiendo los procedimientos de codificación abierta y axial, utilizando para esto el Software cualitativo Atlas ti en su versión siete.

\section{Consideraciones éticas}

Para resguardar la voluntariedad de participación de los entrevistados, se realizaron procesos de consentimiento informado, los cuales culminaron con la lectura y firma de documentos antes de iniciar la producción de los datos. En cuanto al financiamiento del proyecto, este fue desarrollado gracias al Proyecto de Iniciación de Investigación Social en Posgrado (inicio 2014), titulado: "Calidad de vida de familias damnificadas por el terremoto del 27F: Un acercamiento a la vulnerabilidad por desastres socionaturales" de la Facultad de Ciencias Sociales de la Universidad de Chile.

\section{Resultados}

A continuación se presentan los resultados divididos en dos temas principales: el tema uno titulado Nos quedamos con lo puesto: (sobre) viviendo en la incer- 
tidumbre y el tema dos titulado Respuesta social frente al desastre. A su vez, cada tema se subdivide en dos y tres subtemas respectivamente.

Tema 1: Nos quedamos con lo puesto: (sobre)viviendo en la incertidumbre.

El tema "nos quedamos con lo puesto" identifica y describe los significados y acciones llevadas a cabo por las familias afectadas por el desastre ante la etapa de emergencia; a su vez se divide en tres subtemas que enunciamos a continuación. A) Satisfacción de necesidades fisiológicas básicas. Este subtema comprendió los satisfactores bienes materiales o servicios de primera necesidad, así como también quiénes y cómo se obtuvieron dichos satisfactores. B) La pérdida parcial o total de la vivienda orientada al sentido de la significación vivida de la pérdida, sea esta parcial o total, así como las acciones llevadas a cabo para afrontar dicha situación. C) Dios como respuesta de sentido y apoyo para afrontar la crisis. La significación de esta última subcategoría presenta dos sentidos particulares: el primero, como creencia naturalizadora del evento ocurrido, y el segundo, como estrategia de apoyo y afrontamiento frente la emergencia y posterior recuperación. A continuación, las categorías descritas se describen y ejemplifican a través del relato de los afectados.

A) Satisfacción de necesidades fisiológicas básicas: Esta subcategoría remite a la entrega de satisfactores de primera necesidad, tales como alimentos, vestimenta y útiles de aseo. Esto a raíz de la pérdida total o parcial de la vivienda y los enseres materiales post evento. Como respuesta a esta situación, los relatos visibilizan el papel jugado por la sociedad civil, enmarcada no solo a escala territorial-comunal, sino también en un marco amplio de solidaridad de cobertura nacional. Respecto a las redes interpersonales más cercanas a las familias, si bien los vínculos de amistad y por sobre todo los de parentesco son mencionados como redes de apoyo primordial en la entrega de insumos de primera necesidad, para el caso de aquellos que no poseen este tipo redes, se reconoce una amplia generosidad de la comunidad ante esta etapa. Por último, en términos subjetivos, la prolongación temporal de la emergencia se vivencia diferencialmente y se encuentra mediatizada, primordialmente, tanto por las redes de apoyo horizontal con las que se cuenta como por el tipo de tácticas y/o estrategias de afrontamiento utilizadas.

A: Después del terremoto la gente nos apoyó en el sentido de ir a vernos, de cómo estábamos, eh, no sé po, llevarnos algunas cosas que eran necesarios para nosotros, útiles de aseo personales, hartas cosas de apoyo que tuvimos de gente, de gente que sí nos consideraba y, y gente de allá del norte que vino como mi esposo era pescador, eh, vinieron del norte. Vino un amigo del norte a traerles cosas también y no tan solo a nosotros sino a la gente que necesitaba y eso, bueno hartas cosas que uno ha ido, pero es que de repente como que no las recuerdo mucho... Estuvimos casi 3 meses así, recibiendo apoyo de familiares (Mujer 40 años, casada).

B) Nos quedamos sin casa ¿Qué hacemos?: Debido a la pérdida total o parcial de la vivienda, la pregunta que surgió desde los damnificados fue: ¿Y para dónde nos vamos? Frente a esto, los participantes priorizaron la utilización de sus redes sociales o de parentesco, y en caso de no contar con ellas, utilizaron como última opción los campamentos de emergencia o "aldeas" entregadas por el gobierno.

Por otro lado, este cambio sustancial de vivir solos a convertirse en allegados dentro de otro hogar se vivencia como una situación incómoda, invasiva de la privacidad y de la convivencia familiar tanto por el espacio físico-material, al cual se tienen que acondicionar, como por la emergente re-organización hogareña vinculada a los estilos de vida "diferentes" entre los integrantes de las familias que cohabitan el hogar.

En el caso que la familia contara con espacio físico propio, la municipalidad, por medio del MINVU, o instituciones privadas como TECHO, les hizo entrega de viviendas de emergencia para su auto-asentamiento. Sin embargo, si bien esta situación no presenta el grado de incomodidad relacional anteriormente referido, las frágiles condiciones materiales de habitabilidad de la vivienda, así como también de seguridad frente a las condiciones climáticas periódicas (por ejemplo, inundaciones por lluvia), repercuten negativamente en la salud física y mental de los integrantes de las familias.

E: No teníamos a nadie, así que no quedó otra que irse a las aldeas no más (...) era incómodo porque era chiquitita (mediagua entregada) y el espacio era así no má, ahí (indica) así era el espacio como cuarenta centímetros, treinta centímetros. Entonces no había más espacio, entonces era todo incómodo, incómodo para todo. Incómodo todo, porque a veces se dormía, tenía que trabajar en la noche o... era incómodo para todos (Hombre, 30 años, desplazado hacia campamento).

C: Igual fue incómodo también porque es un estilo de vida, cada uno lleva un estilo de vida, o sea, a lo mejor yo me levanto temprano, a lo mejor otra persona no se levanta temprano, otra incomodidad para ello porque una está acostumbrada a otra cosa y no sé po (Mujer, 60 años, desplazado hacia casa de familiares).

C) Dios como explicación y apoyo para afrontar la crisis: La alusión a Dios en los relatos familiares se utiliza bajo dos sentidos: primero, como respuesta de sentido frente al desastre, naturalizando sus causas, acompañada por un sentimiento de incertidumbre y falta de control frente a este tipo de eventos; y segundo, como disposición de acción y fortalecimiento familiar. Esta táctica de afrontamiento ayudó a las familias, en parte, a sobrellevar las consecuencias negativas de la pérdida total o parcial de las condiciones materiales y simbólicas de la (re)producción familiar. A su vez, 
esta creencia espiritual sirvió como motor motivacional para el emprendimiento y esfuerzo familiar e individual en las etapas de normalización, recuperación y reapropiación del proyecto de vida familiar truncado por el desastre.

A: Bueno solo Dios sabe por qué pasan las cosas, él en un momento nos da de todo y de un momento a otro viene $y$ nos arrebata todo de un paraguazo... Se volverá de nuevo a hacer, pero sacar las cosas mejores, bueno uno nunca sabe. Nunca sabe lo que puede ocurrir el día de mañana, uno nunca sabe (Hombre 55 años).

H: Eso porque uno dice que hay, después de todo lo malo viene algo bueno, entonces viene la bendición como uno dice siempre. Eh, uno se fortalece de arriba, no hay nadie más que él que nos fortalezca, a pesar de todas las circunstancias que hemos vivido, que hemos pasado muchas cosas, eh, no hay más más que sobrellevarse, si ¿qué más? ¿Qué más si no hay nadie más que él? (Mujer 52 años).

\section{Tema 2: Desorganización institucional vs apoyo civil.}

El tema 2, "Desorganización institucional vs apoyo civil", describe según lo narrado la valoración del rol que jugaron los distintos actores frente a las etapas de emergencia y recuperación, sean estos instituciones o las propias familias. Este eje, a su vez, se divide en dos subcategorías: A) Respuesta social frente al desastre, o dicho de otro modo, cual fue la capacidad de respuesta percibida y las acciones llevadas a cabo a nivel de instituciones gubernamentales, no gubernamentales y de la sociedad civil no institucionalizada, así como también la evaluación de estas acciones. B) Crisis familiar post desastre, es decir, el impacto percibido del desastre tanto al interior de la estructura como de la organización familiar. Esta segunda subcategoría también incluye las estrategias de afrontamiento desplegadas post emergencia.

A) Respuesta social frente al desastre: Ante el proceso de emergencia, se releva el masivo apoyo voluntario de la sociedad civil, los familiares y las amistades, ejemplificado en el rápido levantamiento de viviendas de emergencia y entrega de satisfactores básicos. En contraposición a esto, se percibe un accionar desorganizado por parte de los organismos gubernamentales. Esta desorganización se traduce en acciones intuitivas y con escasa planificación, ejemplificadas en la falta de transparencia y sobre-intervención en los procedimientos utilizados en el proceso de identificación de damnificados y posterior entrega de beneficios. En cuanto a la etapa de reconstrucción, las familias valorizan negativamente las funciones ejercidas por las instituciones gubernamentales vinculadas al acceso del subsidio habitacional y la entrega definitiva de la vivienda, la cual presenta su traducción psicosocial en desconfianza, confusión y malestar familiar. Este matiz negativo se vincula a la sobre-burocratización percibida por las familias respecto al acceso a la vivienda, el cual sigue los canales tradicionales de postulación de beneficios estatales, no acorde a las condiciones de emergencia propias de una crisis post desastre. A su vez, al comparar la narración de quienes gestionaron su vivienda por medio de un comité de vivienda (EGIS) frente aquellas familias que la realizaron de manera individual, estas últimas manifiestan una mayor presencia de emociones negativas, insatisfacción y conformismo respecto a la demora en la entrega de la casa en comparación a quienes gestionaron la vivienda vía participación barrial.

C: O sea había gente, voluntarios que venían, harta gente
voluntaria, a ayudar, apoyar acá, para hacer las mediaguas
acá, todo, nuestras familias y amigos por sobre todo. Hubo
mucho, mucho apoyo hubo para el sentido de apoyar la
ciudad. Eh, y se paró muy rápido la ciudad, fue muy rápido
que se paró (Hombre 46 años).

E: El gobierno si ayudó pero muy desordenado... Eh, muy desordenado porque aquí traían cosas de todos lados, pero a lo mejor se las entregaban a personas que ya tenían sus cosas y no a la gente que realmente necesitaba, a la gente que estaba trabajando en sus casas (Hombre, 35 años).

A: Sí, fui a obras y había que inscribirse como damnificado. Me fui a obras, me inscribí, hice todo el papeleo. Después con ese papel me fui a SERVIU, tuve que ir a SERVIU. A pesar que, bueno el gobierno pidió un papel que al final pidió, pidió muchos papeles. Porque al final cuando yo me inscribí, el papel de damnificado, ahora piden otro, piden la, la encuesta, ya ahora que ya no pedían... fui muchas veces al SERVIU, después me tomó una señorita y me dieron a elegir tres casas. De las tres casas, ninguna de las tres personas vino a verme, nunca vinieron a verme (Mujer, 45 años).

B) Crisis familiar post-desastre: La familia es la unidad de análisis en la cual se inscriben las tácticas de afrontamiento (o ausencia de estas) frente al desastre, repercutiendo, de manera diferenciada, en el desarrollo de los potenciales proyectos de vida familiar e individual. Bajo este punto de vista, las crisis familiares, debido a la pérdida de la vivienda y a las consecuencias psicosociales que esto conlleva, se inscriben no solo en el plano de las condiciones materiales de habitabilidad previa, sino también en las propias dinámicas familiares y simbólicas que allí circulan. Esta representación y apropiación del espacio personal e íntimo género en las familias sentimientos de incomodidad, inseguridad y desamparo frente a los nuevos cambios no planificados.

Respecto a las tácticas de afrontamiento frente a la crisis, la comunicación y toma de decisiones en conjunto surgen como mecanismos de mantención de la cohesión y organización familiar, así como también la asignación diferenciada de responsabilidad en las tareas cotidianas.

Por último, un aspecto importante a considerar como elemento que mantiene y promueve el bienestar familiar es la mantención del trabajo e ingresos económi- 
cos sostenidos por parte de los y las jefes de hogar. Esta base económica-material sumada a la función social y psicológica del trabajo posibilita la reproducción y mantención de las condiciones de existencia familiar, logrando atenuar el impacto psicosocial de la crisis, en comparación a aquellas familias que perdieron tanto su casa como trabajo, las cuales debieron reestructurar y reorganizar por completo sus condiciones de vida.

A: En la primera casa estábamos todos juntos, estaba el papá, estábamos todos, igual cuesta acostumbrarse un poco a otra casa po... igual las niñitas sobre todo, aunque la Gabi no quería venirse a esta casa, no quería ella, quería seguir en la otra casa aunque no tuviera sus comodidades. $Y$ con el tiempo yo le fui explicando que iba a estar mejor, que ibamos a estar mejor, iba a poder llegar con sus amigas, iba a ser mejor calidad de vida para ella. Después se fue adaptando... (Mujer, 37 años).

C: Mira mi casa que es hermosa (sonríe), eh bueno, gracias a que nos mantuvimos unidos tenemos esto, todo ha sido por el trabajo, por el esfuerzo que cada uno ha tenido. Entonces, eso es bueno porque nadie tiene que decir, eh, yo he he, o no sé po cualquier cosa, pero es todo esfuerzo porque detrás del esfuerzo hay apoyo o tiene sentido que, como familia o sea como somos, esposo, hijos, y apoyarnos unos a otros (Hombre, 35 años).

P: Como familia nos poníamos de acuerdo qué teníamos que hacer para el día a día, mi esposo tenía que salir a trabajar, para poder comprar las cosas para la casa, mi hijo se dedicaba a limpiar la casa y yo iba a toda reunión y cosa que salía... (Mujer, 39 años).

A: Después del tsunami obviamente la mar se echó a perder y no pudimos salir a trabajar... no teníamos con que parar la olla, así que no quedó otra que hacer uno que otro pololito que salía por ahí y apretarse el cinturón no más po... (Hombre, 40 años).

\section{Discusión}

Los estudios que vinculan psicología y desastres han tendido a centrarse en el proceso de emergencia, enfatizando el posible impacto psicosocial de este evento sobre la salud mental de los afectados (Leiva-Bianchi, 2011; López-Ibor, Christodoulou, Maj, Sartorius \& Okasha, 2005). Sin embargo, se torna relevante ampliar el dominio disciplinar de la investigación en psicología bajo la dimensión procesual de la vulnerabilidad, considerando no solo las dimensiones socioculturales de la estructura social, sino también el papel activo y sentido de la subjetividad configurada ante este tipo de eventos (Pérez et al., 2012).

De acuerdo a los resultados de la presente investigación, en la atapa de emergencia surge la primera crisis desde la cual las distintas capacidades de respuesta material, organizacional y simbólica se ven sobrepasadas. En esta etapa, la dimensión de satisfacción de necesidades fisiológicas básicas se presenta como la base mínima necesaria para la subsistencia individual y familiar, donde las acciones desplegadas adoptan una estructura similar al modelo jerárquico piramidal de necesidades de Maslow (1991), bajo la lógica de preservación fisiológica de la vida. Sin embargo, cabe destacar que esta crisis emergente ante el desastre debe ser leída desde el proceso ampliado de la vulnerabilidad social de base, considerando para esto las distintas susceptibilidades y capacidades preventivas, más allá de la contingencia del impacto de la amenaza natural. En consonancia con esto, no es lo mismo significar este evento como una crisis que se suma a la trayectoria cotidiana propia de vulnerabilidad ante los riesgos emergentes continuamente afrontados, sean estos económicos o psicosociales, que significarlo como un evento crítico crucial, el cual marca un antes y un después en la cotidianidad de la persona (Arteaga \& Pérez, 2011).

Por otro lado, en el subtema Nos quedamos sin casa ¿Qué hacemos?, emergen las necesidades de protección e intimidad familiar. En este ámbito, evidenciamos dos tipos de satisfactores. Por un lado, la red de apoyo horizontal compuesta por familiares, amigos y cercanos, y por otro, la red de protección social vertical de tipo gubernamental. En ambas redes, los participantes vivencian el "estar" de allegados o desplazados bajo el sentimiento de incomodidad. No obstante, los grados de mayor malestar, así como la presencia de emociones negativas más frecuentes los expresan aquellas personas que no contaban con redes de apoyo familiar o de amistad. Esto se vincula a la importancia de la presencia y fortalecimiento de las capacidades organizacionales y redes de confianza, provenientes del ámbito familiar, comunitario o institucional, como potenciales recursos colectivos en relación a la anticipación, mitigación y recuperación ante este tipo de eventos.

Otro de los aspectos a considerar de la dimensión subjetiva radica en que la vulnerabilidad se vincula a la representación y apropiación de la vivienda de emergencia, la cual es percibida y valorada de forma negativa. Esto se debe, principalmente, a las reducidas condiciones espaciales de esta, así como también a la pérdida absoluta de intimidad personal y de las prácticas familiares de reproducción cotidiana. Respecto a la salud física, la fragilidad de la infraestructura frente a las cambiantes condiciones climáticas del territorio acrecienta la susceptibilidad a nuevas enfermedades, aumentando aún más la vulnerabilidad de la salud y el presupuesto familiar en este ítem.

Respecto al subtema Dios como explicación y apoyo para afrontar la crisis, este cumple dos funciones subjetivas. En primer lugar, es utilizado como respuesta de sentido y justificación "divina" del desastre bajo el principio de "Sólo Dios sabe por qué hace las cosas"; y en segundo lugar, también es empleado como táctica simbólica-valorica de afrontamiento para el emprendimiento y autogestión personal, sustentada bajo el relato del esfuerzo individual o familiar. En la primera 
función, este "sentido" (sobre)natural del desastre se ve reforzado a su vez por el sentimiento de desamparo hacia las instituciones gubernamentales involucradas, debido principalmente, a la desorganización y desinformación percibida con respecto a estas. Es relevante destacar este aspecto debido a la importancia que juega el papel de un conocimiento organizado en la prevención y preparación frente a los desastres. En esta misma línea, algunas personas todavía no tienen claridad acerca de cómo hacer frente a un potencial peligro de este tipo, a pesar de haber vivido en carne propia el desastre del 27F. Por lo tanto, para generar y fortalecer capacidades de respuestas apropiadas, que vayan más allá del modelo cognitivo-informacional del tradicional modelo institucional de gestión de riesgos, se vuelve relevante establecer un nexo entre el lenguaje técnico de los expertos y el conocimiento popular, buscando así establecer un puente comprensivo que dé paso a una acción planificada por parte de la población.

En cuanto al segundo tema, los participantes manifiestan una valoración diferenciada respecto al rol de los actores involucrados, así como también de las distintas acciones desplegadas por estos. Por un lado, las instituciones gubernamentales presentan una baja valoración por parte de los entrevistados en relación a los mecanismos de identificación de damnificados, los criterios de focalización de recursos y la escasa sensibilidad y planificación ante las necesidades inmediatas y mediatas de la población afectada. En el marco de este punto, en una primera instancia, el Informe Mundial de Desarrollo Humano del año 2014 resalta la importancia de las condiciones estructurales de las naciones ante este tipo de eventos. Como base a estas condiciones, se resalta el papel de un Estado garante de las necesidades de los ciudadanos, lo cual, para el caso de Chile, sería diametralmente opuesto debido al rol subsidiario que este promueve, condicionando diferencialmente las capacidades de respuesta y recuperación de los individuos. En una segunda instancia, se destaca la implementación y utilización de una institucionalidad fundada en la gestión integral del riesgo con énfasis en la reducción de la vulnerabilidad social y no estrictamente, como es el caso chileno, en la mera preparación y respuesta ante las emergencias.

En cuanto a la etapa de recuperación, las instituciones gubernamentales han sostenido la aplicación de procedimientos convencionales en la entrega de bienes y servicios ante situaciones no habituales. Un ejemplo de esto lo constituye el acceso a la vivienda definitiva vía subsidio habitacional. En esta línea, la política de reconstrucción nacional habitacional (Ministerio de Vivienda y Urbanismo, 2010) concebida y desarrollada post desastre comprende al ciudadano solo en su rol consultivo o como meros receptores individuales de subsidios estatales, soslayando y fracturando el capi- tal social comunitario construido por los barrios afectados. Para Bourdieu, capital social se entiende como el "agregado de los recursos reales y potenciales que se vinculan con la posesión de una red duradera de relaciones más o menos institucionalizadas de conocimiento y reconocimiento mutuo" (Bourdieu, 1985). Bajo esta red, las relaciones sociales se basan en la confianza, reciprocidad y cooperación, favoreciendo el beneficio mutuo entre instituciones y sujetos (Putnam, 1995). Por lo tanto, si bien el proceso de reconstrucción habitacional ha avanzado, brindando soluciones familiares e individuales, así como también reparando y levantando la infraestructura pública y privada dañada (Ministerio del Interior y Seguridad Pública, 2014), la mera tarea centrada en la intervención física-material requiere una mirada mucho más amplia y compleja, teniendo como centro los proyectos de vida individuales y colectivos de las comunidades territorializadas.

Por otro lado, las redes horizontales que surgen desde la sociedad civil presentan una evaluación positiva bajo los términos de apoyo social percibido. Estas redes adquieren mayor fuerza pre y post emergencia, a través de acciones vinculadas a la entrega de satisfactores básicos y en el levantamiento de viviendas de emergencia. Terminada la etapa de emergencia, la sociedad civil se repliega, dando paso a los actores institucionales vinculados a la reconstrucción habitacional/publica, así como también a las acciones de recuperación al interior de las familias desplazadas. Respecto a este último actor mencionado, la familia, es el que presenta mayor confianza y credibilidad en los relatos, siendo la base del afecto y apoyo percibido por parte de los entrevistados. La familia, frente a la perdida de las condiciones habituales de producción de existencia, debe optar o por la reorganización y adaptación ante esta nueva situación o el deterioro y desintegración de los vínculos entre sus miembros al no poder hacer frente a la crisis. En consecuencia, tanto las estrategias organizativas como la responsabilidad compartida y la comunicación efectiva se vuelven elementos centrales en la mitigación del daño, en donde el esfuerzo y el trabajo de cada uno de los integrantes de la familia son elementos para "salir adelante" y adaptarse a las nuevas exigencias del entorno. En este sentido, no solo se vuelve necesario (re)configurar las condiciones materiales de existencia, sino también la readecuación del proyecto de vida familiar e individual en conjunto.

Debido a lo anterior, se hace necesario explicitar la estructura espacio-temporal (condiciones previas de vulnerabilidad social, crisis y capacidad de respuesta ante los desastres), resaltando el papel de las condiciones socio-históricas que configuran los distintos riesgos, así como también la capacidad de respuesta y resistencia de las comunidades ante estos. Es así como el terremoto y posterior tsunami del 27F puso de manifiesto la fragilidad territorial respecto a los instru- 
mentos de gestión de riesgo, tales como la actualización de los planes reguladores, la implementación de mapas de riesgos, el registros e identificación de las poblaciones vulnerables o el déficit de urbanización, por mencionar solo algunos. En tanto el proceso de reconstrucción hizo lo suyo respecto a las debilidades institucionales para abordar de forma integral el desarrollo de las localidades afectadas, evidenciando la ausencia de estructuras, normativas, procedimientos, programas, y herramientas ante situaciones excepcionales, como es el caso de los desastres. Por lo tanto, a partir de lo mencionado, los desastres socio-naturales visibilizan las distintas vulnerabilidades de base, así como también la fragilidad institucional para la gestión y manejo adecuado de los riesgos asociados.

A partir de las conclusiones de este trabajo, desde una perspectiva interdisciplinar de estudios en el área, se releva la importancia de una mayor investigación en torno a los elementos subjetivos y sociales, antes, durante y después de los desastres. De acuerdo a lo mencionado con anterioridad, si bien en estos últimos años se han ido incorporando nuevas medidas protocolizadas de respuesta poblacional ante este tipo de eventos y su impacto en la salud mental, sigue siendo notorio el relegado papel que se le ha asignado a la dimensión psicosocial para la comprensión de estos fenómenos. Por último, se hace necesario diseñar e implementar estrategias de intervención comunitaria que contemplen no solo las vulnerabilidades previas y emergentes, sino también el desarrollo de capacidades desde y con las propias personas y comunidades.

\section{Referencias}

Anderson, M. \& Woodrow, P. (1989). Rising from the ashes: development strategies in times of disaster. Boulder: Westview Press.

Arteaga, C. \& Pérez, S. (2011). Experiencias de vulnerabilidad: de las estrategias a las tácticas subjetivas. Universum, 26(2), 67-81. doi:10.4067/S0718-23762011000200004

Arteaga, C., Pérez, S., Castro, F., Fava, D., Molina, G. \& Ramírez, C. (2015). Recursos, estructura de oportunidades y subjetividades en contextos de desastre. Análisis a partir del caso de Chaitén. En A. Arteaga \& R. Tapia (Eds.), Vulnerabilidades y desastres socionaturales (pp. 101-115). Santiago, Chile: Editorial Universitaria.

Banco Interamericano de Desarrollo. (2015). Indicadores de riesgo de desastre y de gestión de riesgos. Programa para América Latina y el Caribe: Chile (Nota técnica IDB-TN-782). Recuperado de http://idbdocs.iadb. org/wsdocs/getdocument.aspx?docnum $=35160011$

Banco Mundial. (2014). Informe sobre el Desarrollo Mundial 2014: Riesgo y oportunidad - La administración del riesgo como instrumento de desarrollo. Recuperado de http://nacionesunidas.org.co/biblioteca/informe-sobre-el-desarrollo-mundial-2014-riesgo-y-oportunidad/
Bourdieu, P. (1985). The social space and the genesis of groups. Theory and society, 14(6), 723-744. Recuperado de https://is.muni.cz/el/1423/podzim2010/ SOC251/um/BOURDIEU The social space and the genesis of groups.pdf

Bresciani, L. (2 de abril de 2010). 1000 pueblos y ciudades que reconstruir. La Tercera. Recuperado de http:// ciudadesintegradas.blogspot.cl/2010/04/1000-ciudades-y-pueblos-que-reconstruir 13.html

Cardona, O. (2003). La necesidad de repensar de manera holística los conceptos de vulnerabilidad y riesgo. "Una Crítica y una Revisión Necesaria para la gestión". Colombia: Red de Estudios Sociales en Prevención de Desastres en América Latina (La RED). Recuperado de http://www.desenredando.org/public/articulos/2003/rmhcvr/rmhcvr may-08-2003.pdf

Cardona, O. (2003). La necesidad de repensar de manera holística los conceptos de vulnerabilidad y riesgo "Una Crítica y una Revisión Necesaria para la Gestión". Centro de Estudios Sobre Desastres y Riesgos. Recuperado de http://www.desenredando.org/public/ articulos/2003/rmhcvr/rmhcvr may-08-2003.pdf

Comisión Económica para América Latina y el Caribe. (2010). Terremoto en Chile. Una primera mirada al 10 de marzo de 2010. Recuperado de http://www.cepal.org/es/ publicaciones/32838-terremoto-chile-primera-mirada-al-10-marzo-2010

Creswell, J., Hanson, W., Plano, V. \& Morales, A. (2007). Qualitative research designs selection and implementation. The counseling psychologist, 35(2), 236-264. doi:10.1177/0011000006287390

Flick, U. (2007). Introducción a la investigación cualitativa. Madrid: Ediciones Morata.

Lavell, A. (noviembre, 2004). Vulnerabilidad Social: una contribución a la especificación de la noción y sobre las necesidades de investigación en pro de la reducción del riesgo. Trabajo presentado en el Seminario Internacional Nuevas Perspectivas en la Investigación Científica y Técnica para la Prevención de Desastres, Lima. Recuperado de http://bvpadindeci.gob.pe/ doc/pdf/esp/doc852/doc852-contenido.pdf

Leiva-Bianchi, M. (2011). Relevancia y prevalencia del estrés post-traumático post-terremoto como problema de salud pública en Constitución, Chile. Revista de salud pública, 13(4), 551-559. Recuperado de http://ref. scielo.org/whybd2

López-lbor, J., Christodoulou, G., Maj, M., Sartorius, N. \& Okasha, A. (Eds.). (2005). Disasters and Mental Health. doi:10.1002/047002125X.fmatter

Maskrey, A. (Comp.). (1993). Los Desastres no son Naturales. Colombia: Tercer Mundo Editores.

Maskrey, A. (1998). El riesgo. En A. Maskrey (Ed.), Navegando entre brumas. La aplicación de los sistemas de información geográfica al análisis de riesgo en América Latina (pp. 9-34). Lima: Red de Estudios Sociales en Prevención de Desastres en América Latina (LA RED). 
Maslow, A. (1991). Motivación y Personalidad. Madrid: Ediciones Díaz de los Santos.

Mendoza, P., Ramos, Y., Ortiz, Ó. \& Jaramillo, J. (2010). Comprensión del significado desde Vygotsky, Bruner y Gergen. Diversitas: Perspectivas en Psicología, 6(1), 37-49. doi:15332/s1794-9998.2010.0001.03

Ministerio de Planificación. (2011). Encuesta Post Terremoto: Principales resultados. Efectos en la calidad de vida de la población afectada por el terremoto/tsunami. Recuperado de http://www.redatam.org/redchl/ mds/encpt/Documentos/informe encuesta_post terremoto.pdf

Ministerio del Interior y Seguridad Pública. (2014). Informe Diagnóstico Estado de la Reconstrucción: Terremoto y Tsunami 27 de febrero del 2010. Recuperado de http:// www.gob.cl/wp-content/uploads/2014/06/Diagnostico-27-f.pdf

Ministerio de Vivienda y Urbanismo (2010). Plan de reconstrucción MINVU: Chile Unido Reconstruye Mejor. Recuperado de http://www.minvu.cl/incjs/download. aspx?glb cod nodo=20100827194336\&hdd nom archivo=Resumen\%20Ejecutivo\%20Plan\%20de\%20 Reconstrucci\%C3\%B3n.pdf

Oficina Nacional de Emergencia del Ministerio del Interior y Seguridad Pública. (Productor). (2014). Estar preparados es tarea de todos [Youtube]. Recuperado de

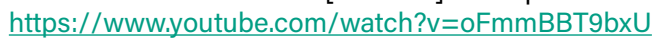

Oliver-Smith, A. (2007). Theorizing vulnerability in a globalized world: a political ecological perspective. En G. Bankoff, G. Frerks \& D. Hilhorst (Eds.), Mapping Vulnerability. Disasters, Development and People (pp. 1024). London: Earthscan.

Pelling, M. (2010). Adaptation to Climate Change: From Resilience to Transformation. London: Routledge.

Pérez, S., Agurto, F. \& Arteaga, C. (2012). Subjetividades frente al riesgo: Un estudio en familias urbanas vulnerables. En C. Zúñiga (Ed.), Psicología, Sociedad y Equidad: Aportes y Desafíos (pp. 109-132). Santiago, Chile: Universidad de Chile.

Programa de las Naciones Unidas para el Desarrollo. (2014). Informe sobre Desarrollo Humano 2014 "Sostener el Progreso Humano: reducir vulnerabilidades y construir resiliencia". Recuperado de http://www.undp.org/ content/undp/es/home/librarypage/hdr/2014-human-development-report.html
Putnam, R. (1995). Bowling alone: America's declining social capital. Journal of democracy, 6(1), 65-78. Recuperado de http://xroads.virginia.edu/ HYPER/DETOC/ assoc/bowling.html

Romero, H. \& Vidal, C. (2015). Exposición, sensibilidad y resiliencia ante los desastres de las ciudades de Concepción-Talcahuano, Chile Central. En A. Arteaga \& R. Tapia (Eds.), Vulnerabilidades y Desastres Socionaturales (pp. 23-39). Santiago, Chile: Editorial Universitaria.

Sandoval, J., Rojas, L., Villalobos, M., Sandoval, C., Aguirre, N., Moraga, F. \& Trujillo, P. (2015). Diagnóstico de capacidades y vulnerabilidades ante el riesgo de desastres socionaturales en brigadas de emergencia de Arica. doi:10.13140/RG.2.1.5046.0246

Strauss, A. \& Corbin, J. (2002): Bases de la investigación cualitativa: Técnicas y procedimientos para desarrollar la teoría fundamentada. Medellín: Universidad de Antioquia.

Taylor, S. \& Bogdan, R. (1998). Introducción a los métodos cualitativos de investigación: La búsqueda de significados. Buenos Aires: Paidós.

Tironi, M. (2014). Disastrous Publics: Counter-enactments in Participatory Experiments. Science, Technology \& Human Values, 40(4), 564-587. doi:10.1177/0162243914560649

Valles, M. (2000). Técnicas cualitativas de investigación social. Madrid: Editorial Síntesis.

Wisner, B. (2016). Vulnerability as Concept, Model, Metric, and Tool. Oxford Research Encyclopedia of Natural Hazard Science. doi:10.1093/acrefore/9780199389407.013.25

Weichselgartner, J. (2001). Disaster mitigation: the concept of vulnerability revisited. Disaster Prevention and Management, 10(2), 85-94. doi:10.1108/09653560110388609 